\title{
An Integral Detection Scheme for Moving Object Indication in Dual-Channel High Resolution Spaceborne SAR Data
}

\author{
Diana Weihing ${ }^{1}$, Stefan Hinz ${ }^{1}$, Franz Meyer ${ }^{2}$, Steffen Suchandt ${ }^{2}$, and Richard Bamler ${ }^{1,2}$ \\ ${ }^{1}$ Remote Sensing Technology \\ Technical University of Munich \\ Arcisstr. 21, 80333 Munich, Germany \\ (Diana.Weihing, Stefan.Hinz)@bv.tum.de \\ ${ }^{2}$ Remote Sensing Technology Institute \\ German Aerospace Center (DLR) \\ Oberpfaffenhofen, 82234 Wessling, Germany \\ (Franz.Meyer, Steffen.Suchandt, Richard.Bamler)@dlr.de
}

\begin{abstract}
Upcoming SAR satellite missions like TerraSAR-X or RADARSAT-2 will deliver high resolution dual channel SAR data with large coverage. These new missions - together with rising interest in area-wide traffic monitoring - motivate spaceborne GMTI as an attractive alternative to conventional traffic data acquisition. However, a moving object appears distorted in the SAR image since the well-known stationary world assumption in the SAR focusing process is violated. In this paper, a detection approach is presented, which considers simultaneously the effects of azimuthal and radial motion of an object. The mathematical framework of this detector combines information of the measured signal, the expected signal, and their variances. Furthermore, the performance of the proposed algorithm is analysed using experimental airborne SAR data.
\end{abstract}

\section{INTRODUCTION}

\section{A. Motivation}

Traffic is one of the major aspects to consider in urban and suburban planning. In order to get information about the traffic on the road network, an observation of large areas is necessary. Usually traffic models are utilized to predict traffic and to forecast transportation, since area-wide acquisition of real traffic data is very expensive with current ground-based systems. Thus, images of spaceborne sensors with a large areal extent are a relatively cheap alternative to collect statistics of traffic on roads to improve traffic models for prediction. With upcoming high resolution spaceborne SAR missions (like TerraSAR-X or Radarsat-2) SAR images up to $1 \mathrm{~m}$ resolution will be available. Additionally, with the Dual Receive Antenna mode, these sensors will provide two SAR images of the same scene within a small timeframe, which enables interferometric measurements for vehicle detection.

\section{B. Related work}

Specific methods for detecting vehicles and estimating their location and motion parameters are required since moving targets suffer from special effects in the SAR processing algorithm. They are not imaged like stationary scatterers, but distorted. The task of detecting cars and estimating the velocity with SAR has been treated in several publications. Depending on the number of apertures, these methods can be grouped into several classes. If more than two channels are available for Ground Moving Target Indication (GMTI), as in most military systems, the use of Space Time Adaptive Processing (STAP) is the optimal method for distinguishing clutter from moving objects [1], [2]. In the case of two-channel systems, like TerraSAR-X or the Canadian Radarsat-2, interferometric approaches can be used for detecting vehicles. Along-Track Interferometry (ATI) [3] and the so-called Displaced Phase Center Array (DPCA) method are the most prominent among them. They can be regarded as an approximation of the limiting case of STAP. For the ATI technique an interferogram needs to be formed from the two SAR images by complex conjugate multiplication. The interferometric phase is related to the object motion. The detection is for instance done based on a constant false alarm rate scheme, where probability density functions depending on the interferometric phase and the amplitude are used [4]. In DPCA processing the two carefully calibrated SAR images are coherently subtracted from each other and the magnitude of the result is evaluated for detection. Hence, the pdfs simplify to a one-dimensional case. These approaches have been extended by integrating a priori information, such as GIS data of road networks, in [5], [6]. In this concept some premises are exploited such as that vehicles are bound to streets, which might not be true in military GMTI. However, these interferometric detection algorithms can only be applied if the motion of the vehicle affects the interferometric phase, which is not the case if vehicles are moving in along-track direction. To estimate ground moving parameters for vehicles travelling in along-track, one method is to apply filterbanks with differently designed matched filters [7], [8].

The presented approach in this paper considers simultaneously the effects in SAR images, which are caused by the vehicle's motion in across- and along-track. The detector scheme is derived from statistical detection theory and its principle relies on comparing an expected signal with the actual measurement. Therefore, different information are combined in this detection algorithm. The expected signal, the measured signal and their variances are included to decide wheter an vehicle is present 
or not.

In the next section a short summary of the different effects moving vehicles cause in SAR images is given. We refer the interested reader to [9], where a comprehensive derivation and review of these effects for spaceborne SAR is given. The proposed detection scheme is explained in Sect. III. Afterwards the performance of this detector is analysed using experimental airborne SAR data (E-SAR from DLR) in Sect. IV and conclusions are drawn in Sect. V.

\section{SAR IMAGING OF MOVING OBJECTS}

In an air- or spaceborne SAR imaging process a radar scans the earth in a side-looking fashion during its flight over the scene. While the sensor is moving it transmits microwave pulses at constant intervals given by the Pulse Repetition Frequency (PRF) and receives the echoes. In Fig. 1 the geometry of an image acquisition is shown. The radar is flying in a certain altitude $h$ along the x-axis, also referred to azimuth direction or along-track. The y-axis, which is oriented perpendicular to the flight path, is usually refered to as ground range or across-track direction. The position of the sensor at a certain point of time is given by $P_{\text {sat }}(t)=$ $\left[x_{\text {sat }}(t), y_{\text {sat }}(t), z_{\text {sat }}(t)\right]$ and the location of the moving target by $P_{\text {target }}=\left[x_{\text {mover }}(t), y_{\text {mover }}(t), z_{\text {mover }}(t)\right]$. The distance between sensor and target corresponds to:

$$
R(t)=P_{\text {sat }}(t)-P_{\text {target }}(t)
$$

Herewith, $R_{0}=\sqrt{y_{0}^{2}+H^{2}}$ is the distance at $t=0$ (the shortest range between SAR and target).

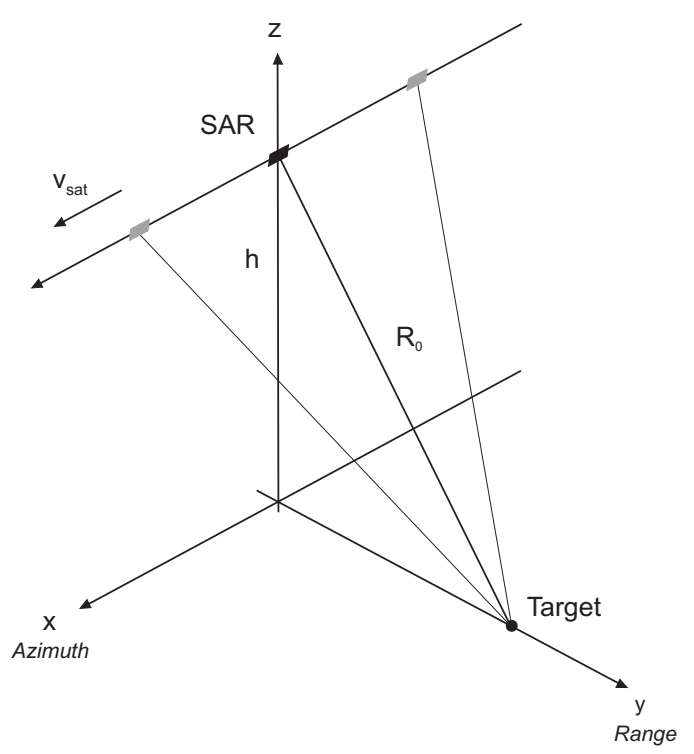

Fig. 1. Imaging geometry of a spaceborne SAR

The received echo is a replica of the transmitted signal backscattered from the ground. For a detailed representation of the echo signal see [10]. Here the received echo signal is simplified without considering range cell migration and can be written as:

$$
h(t)=\exp \left(-j \frac{4 \pi}{\lambda} R(t)\right)=\exp \left(j \pi F M t^{2}\right)
$$

where $F M=-\frac{2}{\lambda} \frac{v_{s a t} v_{b}}{R_{0}}$ is the frequency modulation rate of the azimuth chirp.

To form a SAR image out of the received echoes a matched filter algorithm is applied. The filter for azimuth focusing is defined by:

$$
s(t)=\exp \left(-j \pi F M t^{2}\right)
$$

To circumvent an extensive convolution in time-domain, the operation is done in the frequency-domain by multiplicating the spectra of echos signal and filter.

$$
U(f)=H(f) \cdot S(f)
$$

However, for this process a stationary-world matched filter is assumed. Significantly moving and even accelerating objects violate this assumption and therefore the target will not be imaged "correctly". The dependencies on object velocity and acceleration components can be seen when expanding the range equation $R(t)$ into a third order Taylor series:

$$
\begin{aligned}
& R(t)=R_{0}+\frac{y_{0} v_{y_{0}}}{R_{0}} t+ \\
& \frac{1}{2 R_{0}}\left[\left(v_{x_{0}}-v_{s a t}\right)^{2}+v_{y_{0}}^{2}\left(1-\frac{y_{0}^{2}}{R_{0}^{2}}\right)+y_{0} a_{y_{0}}\right] t^{2}+ \\
& \frac{1}{2 R_{0}}\left[v_{y_{0}} a_{y_{0}}\left(1-\frac{y_{0}^{2}}{R_{0}^{2}}\right)+\left(v_{x_{0}}-v_{\text {sat }}\right) a_{x_{0}}\right] t^{3}- \\
& \frac{1}{2 R_{0}}\left[\frac{y_{0} v_{y_{0}}\left(v_{x_{0}}-v_{s a t}\right)^{2}+y_{0} v_{y_{0}}^{3}}{R_{0}^{2}}\right] t^{3}
\end{aligned}
$$

where $v_{x_{0}}$ and $v_{y_{0}}$ are the components of target's velocity in along- or across-track, respectively, and $a_{x_{0}}$ and $a_{y_{0}}$ the accelerations in these directions, each at the time $t=0$. The imaging effects caused by the uncompensated motion parameters are explained in the following Sections.

\section{A. Motion in across-track}

The object is assumed to move in across-track with a constant velocity. This results in a linear change of the range history, so that the shortest distance is not at time $t=0$ anymore, is shifted depending on the velocity in line-of-sight $v_{\text {los }}=v_{y_{0}} \cdot \sin (\theta)$, with $\theta$ being the local elevation angle. Therefore, the echo signal of the moving target can be written as:

$$
h_{m t_{\text {across }}}(t)=\exp \left(j \pi F M t^{2}\right) \cdot \exp \left(-j \frac{4 \pi}{\lambda} v_{l o s} t\right)
$$

and the spectrum of the moving target after focusing with a stationary-world matched filter is finally:

$U_{m t_{\text {across }}}(f)=\exp \left(-j 2 \pi \frac{2}{\lambda F M} f v_{\text {los }}\right) \exp \left(-j 2 \pi \frac{2}{\lambda^{2} F M} v_{\text {los }}^{2}\right)$ 
According to the correspondencies of the Fourier Transform the linear phase causes a shift in time domain. The shift in time-domain is:

$$
t_{\text {shift }}=\frac{2 v_{l} \text { os }}{\lambda F M}
$$

and can also be expressed in meters:

$$
\Delta_{\text {azimuth }}=-R \frac{v_{\text {los }}}{v_{\text {sat }}}
$$

This means the vehicle gets displaced in the image in azimuth direction depending on its line-of-sight velocity.

\section{B. Motion in along-track}

Let us consider the case the vehicle is moving in azimuth direction with constant velocity. Along-track motion changes the relative velocity between sensor and scatterer $\left(v_{x_{0}}-v_{\text {sat }}\right)$ (cf. Equ. (5)) leading to a change of the quadratic part of the range history. A change of the quadratic component corresponds to a change of the FM rate according to:

$$
\begin{aligned}
F M_{m t} & =-\frac{2}{\lambda R}\left(\sqrt{v_{s a t} v_{B}}-\sqrt{\frac{v_{\text {sat }}}{v_{B}}} v_{x 0}\right)^{2} \\
& =F M\left(1-\frac{v_{x 0}}{v_{B}}\right)^{2}
\end{aligned}
$$

With the new FM rate $F M_{m t}$ the echo signal results in:

$$
h_{m t_{\text {along }}}(t)=\exp \left(j \pi F M_{m t} t^{2}\right)
$$

Focusing $h_{m t_{\text {along }}}(t)$ with a stationary world matched filter (SWMF) does not fully compensate for the quadratic phase term leading to a spread of the received signal corresponding to:

$$
U_{m t_{\text {along }}}(f)=\exp \left(-j \pi \frac{f^{2}}{\delta F M}\right)
$$

where $\frac{1}{\delta F M} \approx-\lambda \sqrt{\frac{v_{s a t}}{v_{B}}} \frac{v_{x 0}}{\left(v_{s a t} v_{B}\right)^{\frac{3}{2}}} R$. Considering the stationary phase approximation the width of the focused peak can be approximated by:

$$
\Delta t \approx \frac{P R F}{\delta F M}=2 T_{A} \frac{v_{x 0}}{v_{B}}
$$

Interpretating this equation shows that a target gets smeared twice the way it moved during the time of illumination $T_{A}$.

\section{Acceleration in across-track}

Like the component of along-track motion, acceleration in across-track mainly affects the quadratic part of Equ. (5). Hence, the acceleration in range also causes a spreading of the energy and the target will be smeared in azimuth. The crosstalk of along-track motion and across-track acceleration hampers the interpretability of along-track smearing without having additional information available.

\section{Acceleration in along-track}

Acceleration in along-track appears in the cubic term of the range history (cf. Equ. (5)), which causes an assimetry of the focused point spread function. However, for TerrSAR-X these effects are neglible [9].

The effects caused by across-track and along-track motion as well as across-track accelaration reach significant values when plugging the TerraSAR-X instrument parameters into the above formulae. We refer the reader to [6] for a detailed analysis of these effects regarding TerraSAR-X.

\section{Proposed Likelihood Ratio Detector}

As shown in Sec. II a moving object causes an interferometric phase, hence also a displacement, and it causes a blurring of its signal. However, with the knowledge of roads and their directions, it is possible to forecast a signal one expects for certain situations. To decide whether a vehicle is existent or not, these signals will be compared with the actual value in the SAR image. Assuming the existence of a vehicle, an expected signal hidden in clutter is compared with the actual measurement.

The mathematical framework for this proposed approach is derived from statistical detection theory. Two hypotheses $H_{0}$ and $H_{1}$ are defined:

$H_{0}$ : only clutter and noise are existent

$H_{1}$ : signal plus clutter and noise are existent

Together with the corresponding probability density functions $f\left(\vec{x} \mid H_{0}\right)$ and $f\left(\vec{x} \mid H_{1}\right)$, these hypotheses allow setting up a likelihood ratio test given by:

$$
\Lambda=\frac{f\left(\vec{x} \mid H_{1}\right)}{f\left(\vec{x} \mid H_{0}\right)}
$$

where

$$
f\left(\vec{x} \mid H_{0}\right)=\frac{1}{\pi^{2}|C|} \exp \left\{-\vec{X}^{H} C^{-1} \vec{X}\right\}
$$

and

$$
f\left(\vec{x} \mid H_{1}\right)=\frac{1}{\pi^{2}|C|} \exp \left\{-(\vec{X}-\vec{S})^{H} C^{-1}(\vec{X}-\vec{S})\right\}_{(16)}
$$

are circular Gaussian Random Processes with $\vec{S}$ being the expected signal, $\vec{X}$ being the measured signal, $C$ being the covariance matrix, and $(*)^{H}$ being the Hermitian Matrix. See e.g. [11] for their derivation.

Finally, from Eqs. 14-16 we can derive the Bayesian decision rule of log-likelihood test, which computes to:

$$
\left|\vec{S}^{H} C^{-1} \vec{X}\right|>\alpha
$$

which is the mathematical framework for the detection scheme.

In particular, following pieces of information are combined in this detection approach: the expected signal $\vec{S}$, the measured signal $\vec{X}$, as well as their covariances $C$, which are described in more detail below.

The measured signal consists of the SAR images from the two apertures: 


$$
\vec{X}=\left(\begin{array}{c}
X_{1} \\
X_{2}
\end{array}\right)
$$

with the indices standing for the two channels

For the expected signal, a priori knowledge is required. With the information of road location and road direction from GIS data an expected phase for each pixel depending on the pixel's distance to the corresponding road segment can be forecasted. With the expected phase $\phi$ the expected signal can be derived:

$$
\vec{S}=\left(\begin{array}{c}
S_{1} \\
S_{2}
\end{array}\right)=\left(\begin{array}{c}
\exp \left\{j \frac{\phi}{2}\right\} \\
\exp \left\{-j \frac{\phi}{2}\right\}
\end{array}\right)
$$

where also the indices significate the two channels. The covariance matrix is defined as in [11]:

$$
C=E\left\{X X^{H}\right\}=\left(\begin{array}{cc}
\bar{I}_{1} & \gamma \bar{I} \\
\gamma^{*} \bar{I} & \bar{I}_{2}
\end{array}\right) \approx \bar{I}\left(\begin{array}{ll}
1 & \gamma \\
\gamma & 1
\end{array}\right)
$$

with $\bar{I}=\sqrt{\bar{I}_{1} \bar{I}_{2}}=\sqrt{E\left[\left|u_{1}\right|^{2}\right] E\left[\left|u_{2}\right|^{2}\right]}$

However in real scenarios with roads heading in any direction not only an interferometric phase occurs, but also a blurring of the signal over several resolution cells. Thus, not only a single pixel has to be compared with its expected value. Neighbouring pixels are included into the mathematical framework to decide whether the considered pixel is a vehicle or not. The possible blurring effect in these adjacent pixels subject to existing along-track motion or across-track acceleration will be adjusted by variation of filters to obtain a focused object and thus an optimized peak-to-sidelobe-ratio for its signal. Therefore, the input into the detection scheme looks like:

$$
\begin{aligned}
& \vec{X}=\left(\begin{array}{c}
X_{1,1} \\
\vdots \\
X_{1, n} \\
X_{2,1} \\
\vdots \\
X_{2, n}
\end{array}\right), \vec{S}=\left(\begin{array}{c}
S_{1,1} \\
\vdots \\
S_{1, n} \\
S_{2,1} \\
\vdots \\
S_{2, n}
\end{array}\right) \\
& C=\left(\begin{array}{cccccc}
1 & & 0 & \gamma & & 0 \\
& \ddots & & & \ddots & \\
0 & & 1 & 0 & & \gamma \\
\gamma & & 0 & 1 & & 0 \\
& \ddots & & & \ddots & \\
0 & & \gamma & 0 & & 1
\end{array}\right)
\end{aligned}
$$

The complex pulse responses, actual and forecasted, are characterized by $\vec{X}_{1, i}$ and $\vec{S}_{1, i}$ for image 1 and $\vec{X}_{2, i}$ and $\vec{S}_{2, i}$ for image 2. These signals are blurred in azimuth over the cells $i$ : 1 - $n$. Thereby, a reasonable guess for $n$ can be derived from Equ. 13.

Until now the proposed likelihood ratio detector is realized for the case of considering only one expected value with the actual measurement. Adjacent pixels to compensate for the blurring effect are not yet included into the decision process. Hence, the following analyses are initial results for this detector. The implementation of the module that includes possible blurring effects is in progress.

\section{RESUlTS}

In prior work of our group, a modular traffic processor has already been developed which processes SAR data specifically with the objective of moving vehicle detection [12], [13]. Different detectors (ATI and DPCA) are integrated for finding vehicles and can be selected individually or can be combined. The proposed detection algorithm has been included into this environment so that a comparison of the individual methods is possible.

The detection approach is tested on real SAR data. DLR's E-SAR data of a flight campaign near DLR's Oberpfaffenhofen campus has been used. One sees in Fig. 2 a part of the motorway A96 heading nearly in across-track. The detected vehicles at their displaced positions are marked with red rectangles. The triangels are the positions of these vehicles backprojected to the assigned road. These triangles are colorcoded regarding their estimated velocity. In all figures, the range is from blue to red, standing for 0 to $100 \mathrm{~km} / \mathrm{h}$.

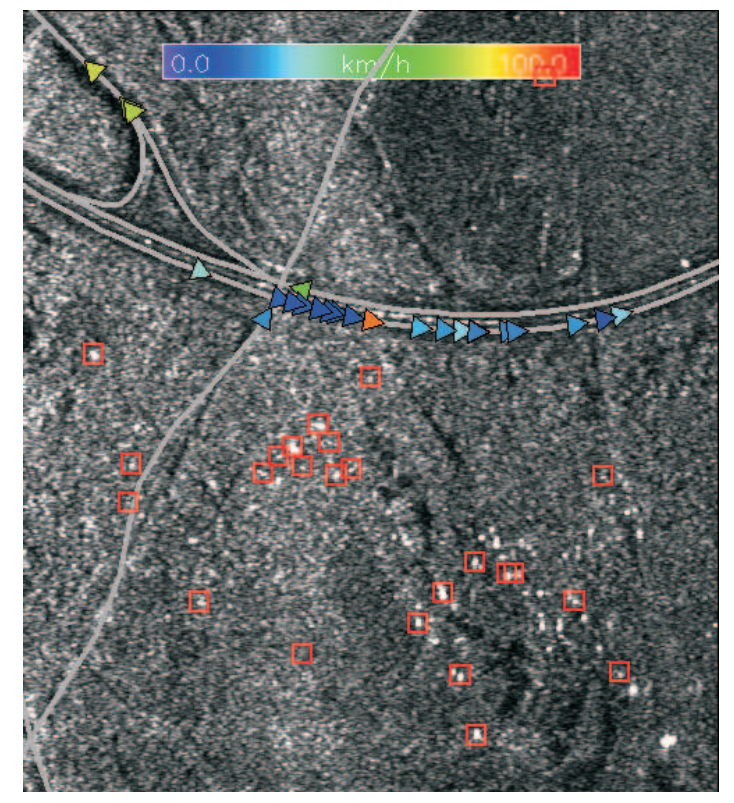

Fig. 2. Detection results using the proposed likelihood ratio detector

During data acquisition there was a traffic jam with a median velocity of about $17 \mathrm{~km} / \mathrm{h}$ in this section. Ignoring the orange-coded object, which is obviously a false detection, a median velocity from the detections can be calculated of $18 \mathrm{~km} / \mathrm{h}$, which approximates very well the actual median velocity of this traffic jam. Ignoring such an outlier is valid in case of traffic congestion, since vehicle velocities in queues depend on each other spatially.

To evaluate the performance of the likelihood ratio detector, its results are compared to the detection results using only ATI or DPCA, respectively. Therefore, the different detection algorithms were used separately and their results are shown in Fig. 3 and Fig. 4. 


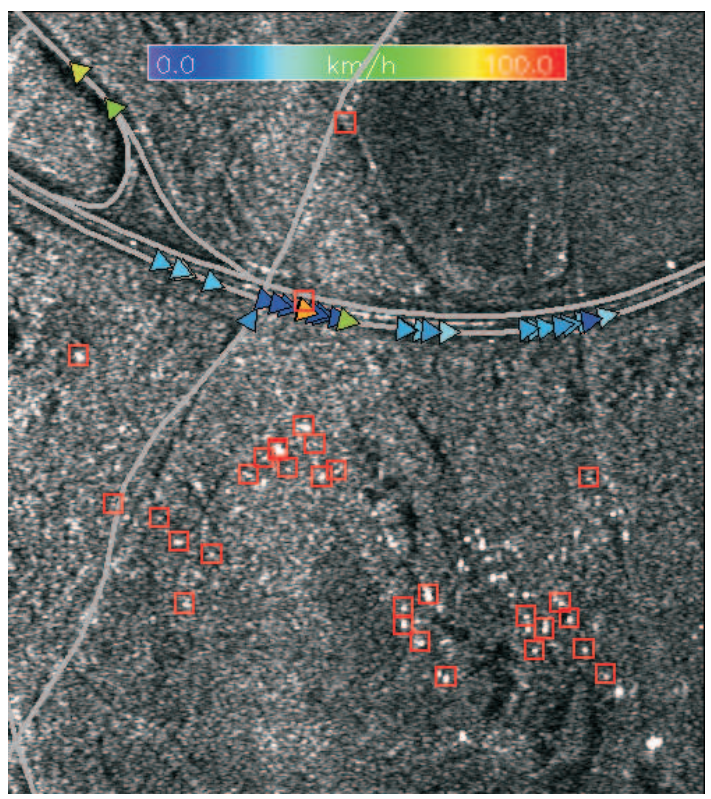

Fig. 3. Detection results using DPCA

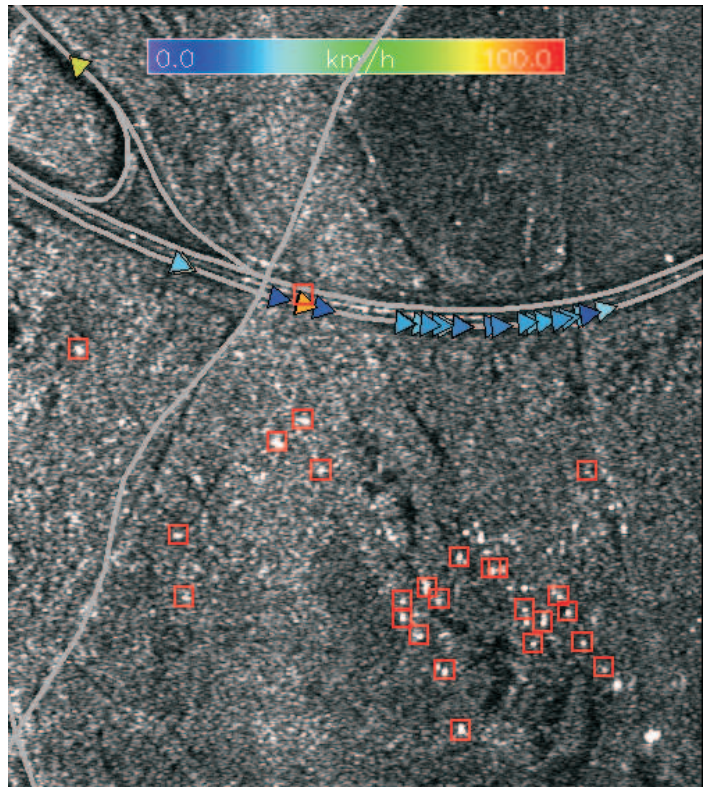

Fig. 4. Detection results using ATI

In all cases, i.e. in all of these three detection schemes, fixed values for the decision thresholds have been used. These thresholds affect of course the detection rate and the false alarm rate. Here reasonable values have been chosen for the detections.

A comparison between the detection results regarding the proposed detection approach is shown in Tab. I. The number of detected vehicles, which have been detected redundantly with different detectors, are listed there.

Most of the detections were achieved with all three detectors. However, a few were detected only with the new
TABLE I

REDUNDANT DETECTIONS ACHIEVED USING DIFFERENT DETECTORS

\begin{tabular}{|l|c|}
\hline Detected with: & Number of detected vehicles \\
\hline likelihood ratio detector & 3 \\
\hline likelihood ratio detector and DPCA & 6 \\
\hline likelihood ratio detector and ATI & 4 \\
\hline likelihood ratio detector, DPCA, and ATI & 11 \\
\hline
\end{tabular}

detection approach. The redundant detections allow to rate a higher probability of being correctly estimated. With "single" detections one migth conclude a rise of the probability of being a false detection.

Therefore, it should be noted, that this detectors are not compared being competetive, but complementary. If the results are combined together a greater set of detections can be achieved and the results can support each other. Candidates found with more detectors have thus a higher probability of being a correctly detected vehicle.

Having these detections projected back onto the road axis as in Fig. 2 - Fig. 4, it is possible to derive parameters for the situation on the road and using them for traffic models, see e.g. [12].

\section{CONCLUSION}

The results show that it is possible to detect vehicles with the proposed likelihood ratio detector and that the result is comparable with those results received with conventional detection schemes. However, one must note that these detectors don't have to be competetive but complementary to support redundant detections and therefore to verify the results. With the enhancement, being in progress, of considering adjacent pixels in the decision process of the proposed detector one may expect additional improvements for the results.

\section{ACKNOWLEDGMENT}

Special thanks go to the DLR traffic processor developers for their support and for providing the programs to make analyses of the proposed detection scheme.

\section{REFERENCES}

[1] J. Ender, "Space-time processing for multichannel synthetic aperture radar," Electronics \& Communication Engineering Journal, vol. 11, no. 1, pp. 29-38, 1999.

[2] R. Klemm, Space-time adaptive processing, R. Klemm, Ed. London, United Kingdom: The Institute of Electrical Engineers, 1998.

[3] I. Sikaneta and C. Gierull, "Two-Channel SAR Ground Moving Target Indication for Traffic Monitoring in Urban Terrain," in International Archives of Photogrammetry, Remote Sensing and Spatial Information Sciences, vol. 36, no. 3/W24, 2005, pp. 95-101.

4] C. Gierull, "Moving Target Detection with Along-Track SAR Interferometry," Defence Research \& Development Canada, Tech. Rep. DRDCOTTAWA-TR-2002-084, 2002.

[5] F. Meyer, S. Hinz, A. Laika, and R. Bamler, "A-priori Information Driven Detection of Moving Objects for Traffic Monitoring by Spaceborne SAR," in International Archives of Photogrammetry, Remote Sensing, and Spatial Information Sciences, vol. 36-3W/24, 2005, pp. 89-94.

[6] F. Meyer, S. Hinz, A. Laika, D. Weihing, and R. Bamler, "Performance analysis of the terrasar-x traffic monitoring concept," ISPRS Journal of Photogrammetry and Remote Sensing, vol. 61, no. 3-4, pp. 225-242, 2006.

[7] C. Gierull and I. Sikaneta, "Ground Moving Target Parameter Estimation for Two-Channel SAR," in EUSAR 2004 Conference. Ulm, Germany: on CD, 2004. 
[8] D. Weihing, S. Hinz, F. Meyer, A. Laika, and R. Bamler, "Detection of along-track ground moving targets in high resolution spaceborne SAR images," in International Archives of Photogrammetry, Remote Sensing, and Spatial Information Sciences, vol. 36-7, 2006.

[9] S. Hinz, F. Meyer, M. Eineder, and R. Bamler, "Traffic Monitoring with spaceborne SAR - Theory, simulations, and experiments," in Computer Vision and Image Understanding, to appear, 2007.

[10] R. Bamler and B. Schättler, SAR geocoding. Karlsruhe: Wichmann, 1993, ch. 3, pp. 53-102.

[11] R. Bamler and P. Hartl, "Synthetic aperture radar interferometry," Inverse Problems, pp. R1-R54, 1998.

[12] S. Suchandt, M. Eineder, R. Müller, A. Laika, S. Hinz, F. Meyer, and G. Palubinskas, "Development of a GMTI processing system for the extraction of traffic information from TerraSAR-X data," in EUSAR 2006 Conference, on CD, Ed., 2006

[13] S. Suchandt, M. Eineder, H. Breit, and H. Runge, "Analysis of ground moving objects using SRTM / X-SAR data" ISPRS Journal of Photogrammetry \& Remote Sensing, vol. 61, no. 3-4, pp. 209-224, 2006. 